\title{
Estimating Constrained Multi-fiber Diffusion MR Volumes by Orientation Clustering
}

\author{
Ryan P. Cabeen ${ }^{1}$, Mark E. Bastin ${ }^{2}$, and David H. Laidlaw ${ }^{1}$ \\ 1 Computer Science Department, Brown University, Providence, RI, USA \\ \{cabeen, dhl\}@cs. brown.edu \\ 2 Centre for Clinical Brain Sciences, University of Edinburgh, Edinburgh, UK \\ Mark.Bastin@ed.ac.uk
}

\begin{abstract}
Diffusion MRI is a valuable tool for mapping tissue microstructure; however, multi-fiber models present challenges to image analysis operations. In this paper, we present a method for estimating models for such operations by clustering fiber orientations. Our approach is applied to ball-and-stick diffusion models, which include an isotropic tensor and multiple sticks encoding fiber volume and orientation. We consider operations which can be generalized to a weighted combination of fibers and present a method for representing such combinations with a mixture-of-Watsons model, learning its parameters by Expectation Maximization. We evaluate this approach with two experiments. First, we show it is effective for filtering in the presence of synthetic noise. Second, we demonstrate interpolation and averaging by construction of a tractography atlas, showing improved reconstruction of white matter pathways. These experiments indicate that our method is useful in estimating multi-fiber ball-and-stick diffusion volumes resulting from a range of image analysis operations.
\end{abstract}

Keywords: diffusion imaging, tractography, atlasing, multi-fiber models, white matter, ball-and-stick, filtering, interpolation.

\section{Introduction}

Diffusion magnetic resonance imaging enables quantitative mapping of tissue microstructure properties. This modality is especially valuable for studying white matter in the human brain, as water molecule diffusion models enable both local measurement of fiber integrity as well as more global reconstruction of white matter structure through tractography. While these are rich sources of information, multi-direction diffusion models pose a challenge for common operations, such as filtering, interpolation, and averaging. This is due to the directional nature of fiber models and the correspondence problem of matching fibers between voxels [1. This is a major concern for atlas-based neuroimaging studies, where these operations are used to spatially normalize a population and perform statistical analysis.

Our contribution is the presentation and evaluation of an approach for filtering, interpolating, and averaging ball-and-stick fiber models, a multi-compartment 
water diffusion model that has relatively few parameters and is commonly used for tractography [2]. Our approach bears most similarity to a method for interpolating multi-tensor models by Taquet, et al. 3. While the ball-and-stick model consists of tensor compartments, they are not positive definite, so the Gaussian mixture simplification approach proposed in that work is not well defined for this constrained model. Instead, we employ the mixture-of-Watsons model proposed for fiber modeling by Rathi el al [4, present an efficient Expectation Maximization algorithm for learning its parameters from weighted samples, and evaluate its applications with two experiments. The Watson distribution has been notably used to model fibers in several other works, including characterizing orientation error [5], performing filtered tractography [6], statistical testing [7], and atlas space averaging [ $]$.

In the following section, we first review the ball-and-stick diffusion model, then show how mixture-of-Watsons clustering can be used to estimate multi-fiber volumes, followed by a description of an Expectation Maximization algorithm to efficiently learn the model parameters. We then present results from two experiments: the first tests the performance of mixture-of-Watsons filtering in the presence of synthetic noise, comparing to the heuristic discussed by Taquet, et al. [3] the second demonstrates the construction and virtual dissection of a tractography atlas, comparing it to the single tensor and heuristic reconstructions. We end with a discussion and concluding remarks.

\section{Methods}

\subsection{Diffusion Model}

A common multi-fiber model consists of a linear combination of tensors. The predicted signal $S_{i}$ of the $i$-th volume for such a model is:

$$
S_{i}=S_{0} \sum_{j=0}^{M} f_{j} \exp \left(-b_{i} \boldsymbol{g}_{i}^{T} \mathbf{D}_{j} \boldsymbol{g}_{i}\right)
$$

given M fiber compartments, gradient encoding direction $\boldsymbol{g}_{i}$, b-value $b_{i}$, unweighted signal $S_{0}$, fiber volume fraction $0 \leq f_{j} \leq 1$, and $\sum_{j=0}^{M} f_{k}=1$. In this paper, we consider the ball-and-stick variety of this model, which is constrained to have a completely isotropic first component $\mathbf{D}_{0}=\operatorname{diag}(d, d, d)$ and completely anisotropic subsequent components $\mathbf{D}_{j}=d \boldsymbol{v}_{j} \boldsymbol{v}_{j}^{T}$, given diffusivity $d$ and fiber orientation $\boldsymbol{v}_{j}$. The anisotropic tensor is given by the outer-product of the fiber orientation vector, whose eigenvalues are $\{1,0,0\}$. It should be noted that this tensor is not positive definite, thus Riemannian tensor manifold methods and the Burg matrix divergence [3] are not well defined.

\subsection{Mixture-of-Watson Estimation}

In this section, we describe our method for estimating ball-and-stick models with mixture-of-Watson clustering. We take an approach similar to [3] and 
consider operations which can be generalized as weighted combinations of fiber compartments. Examples include per-voxel averaging across a population, trilinear interpolation, and Gaussian filtering. For simplicity, we use set notation to represent combinations of fibers. A single voxel model can be described by a volume-weighted combination $S_{i}=\bigcup_{j}^{N_{i}}\left(f_{i j}, \boldsymbol{v}_{i j}\right)$, and a weighted combination of all grouped voxel models $G$ can then be defined by the combination of those voxel-wise models, where each resulting weight is the product of the voxel volume fraction $f_{i j}$ and the per-model weight $s_{i}$ :

$$
G=\bigcup_{i}^{M}\left(s_{i}, S_{i}\right)=\bigcup_{i}^{M} \bigcup_{j}^{N_{i}}\left(s_{i} f_{i j}, \boldsymbol{v}_{i j}\right)=\bigcup_{k}^{K}\left(w_{k}, \boldsymbol{v}_{k}\right)
$$

where $K=\sum_{i}^{M} N_{i}$, and $k$ indexes $(i, j)$, i.e. $w_{k}=s_{i} f_{i j}$ and $\boldsymbol{v}_{k}=\boldsymbol{v}_{i j}$.

This weighted combination alone offers one solution to operations such as averaging and interpolating, but the result is overly complex. Taquet, et al. [3] have proposed Gaussian mixture simplification as an approach to reduce this complexity for the case of multi-tensor models; however, this method is not well-defined for the stick models, as previously explained. Our approach offers a similar clustering-based solution by modeling the distribution of fibers by a mixture-of-Watsons, which was suggested for multi-fiber modeling [4]. The Watson distribution [9] is a model for directional statistics that was proposed for single fiber modeling by Cook, et al. 5] and bears similarity to a Gaussian distribution. The space of directions can be modeled as points on the $S^{2}$ with antipodal equivalence, i.e. $\left\{\boldsymbol{v} \in \mathbb{R}^{3}:\|\boldsymbol{v}\|=1\right.$ and $\left.\boldsymbol{v} \sim-\boldsymbol{v}\right\}$. Its probability density, given concentration $\kappa$ is then:

$$
W(\boldsymbol{v} ; \theta)=A(\kappa) \exp \left(\kappa\left(\boldsymbol{\mu}^{T} \boldsymbol{v}\right)^{2}\right)
$$

for model parameters $\theta=(\boldsymbol{\mu}, \kappa)$ and normalization constant $A(\kappa)=M\left(\frac{1}{2}, \frac{3}{2}, \kappa\right)^{-1}$ given by the confluent hyper-geometric function, sometimes known as ${ }_{1} F_{1}$ or the Kummer function. The density of the set of fibers $G$ can then be modeled by a mixture of $C$ Watson distributions:

$$
p(\boldsymbol{v} ; \Theta)=\sum_{c=1}^{C} \alpha_{c} W\left(\boldsymbol{v} ; \theta_{c}\right)
$$

given mixture model parameters $\Theta=\left(\alpha_{1}, \theta_{1}, \ldots, \alpha_{C}, \theta_{C}\right)$ and $\sum \alpha_{c}=1$. The number of components $C$ controls the resulting complexity and may be chosen by some method of model selection, which is not explored here. We use a weighted samples version of the Expectation Maximization algorithm presented by Sra, et al. [10] to learn the parameters. The assignment and update steps are described as follows. 
E-Step: Assign responsibility of each component $c$ for each fiber $k$

$$
\pi_{k c}=p\left(c \mid \boldsymbol{v}_{k}, \Theta\right)=\frac{\alpha_{c} W\left(\boldsymbol{v}_{k} \mid \theta_{c}\right)}{\sum_{c}^{C} \alpha_{c} W\left(\boldsymbol{v}_{k} \mid \theta_{c}\right)}
$$

M-Step: Update the model parameters for each component $c$

$$
\begin{aligned}
\alpha_{c} & =\frac{\sum_{k}^{K} w_{k} \pi_{k c}}{\sum_{k}^{K} w_{k}} \\
\boldsymbol{\mu}_{c} & =\frac{\sum_{k}^{K} w_{k} \pi_{k c} \boldsymbol{v}_{k} \boldsymbol{v}_{k}^{T} \boldsymbol{\mu}_{c}}{\left\|\sum_{k}^{K} w_{k} \pi_{k c} \boldsymbol{v}_{k} \boldsymbol{v}_{k}^{T} \boldsymbol{\mu}_{c}\right\|} \\
\kappa_{c} & =\frac{\sum_{k}^{K} w_{k} \pi_{k c}}{\sum_{k}^{K} w_{k} \pi_{k c}\left(1-\left(\boldsymbol{\mu}_{c}^{T} \boldsymbol{v}_{k}\right)^{2}\right)}
\end{aligned}
$$

where $\kappa$ and $A(\kappa)=\frac{\kappa}{\pi \exp (\kappa)}$ are found similarly to Schwartzman, et al. [7]. This process can also be adapted to a "k-means" algorithm, similar to diametrical clustering, with hard assignment in the E-step and fixed parameters in the Mstep. The k-means approach is initialized from a set of randomly selected fibers, and the Expectation Maxmization-approach is initialized with the k-means solution. The resulting clustering is then used to estimate a simpler fiber model $\hat{G}=\bigcup_{c}^{C}\left(\alpha_{c}, \boldsymbol{\mu}_{c}\right)$, by assigning the mixing parameters to volume fractions and component means to fiber orientations. We compare to a "heuristic" method described in [3. This heuristic makes a simplifying assumption that fiber compartments are matched across voxels by volume-fraction rank, as opposed to the proposed matching defined by clustering. For example, when averaging twodirection models, the smaller volume compartments would be averaged separately from the larger volume compartments.

\section{Experiments and Results}

\subsection{Data Acquisition and Processing}

Diffusion volumes were acquired from 80 healthy volunteers uniformly distributed in age from 25 to 64 years with 39 male subjects following an IRB-approved protocol. Imaging was conducted on a GE $1.5 \mathrm{~T}$ scanner with a voxel size of $2 \mathrm{~mm}$ x $2 \mathrm{~mm}$ x $2 \mathrm{~mm}$, matrix size $128 \times 128$, and 72 contiguous axial slices. For each subject, a total of 71 volumes were acquired, with seven $\mathrm{T}_{2}$-weighted volumes (b-value $0 \mathrm{~s} / \mathrm{mm}^{2}$ ) and 64 diffusion-weighted volumes (b-value $1000 \mathrm{~s} / \mathrm{mm}^{2}$ ) and distinct gradient encoding directions. All diffusion MRI data were corrected for motion and eddy current artifacts by affine registration to the first $\mathrm{T}_{2}$-weighted volume using FSL Flirt (http://www.fmrib.ox.ac.uk) with a mutual information cost function. The gradient encoding directions were rotated to account for the alignment, and non-brain tissue was removed using FSL Bet. Single tensor models were fit using FSL DTIfit, and two-direction ball-and-stick diffusion models were fit using FSL Xfibres. All subjects were mapped to a population-specific template by tensor registration using DTI-TK [11. 


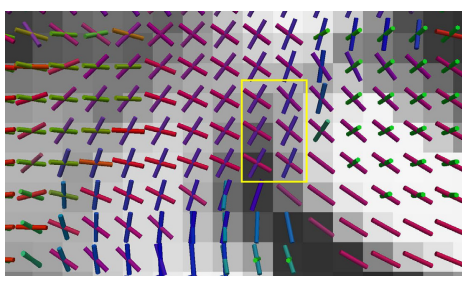

(a) Source

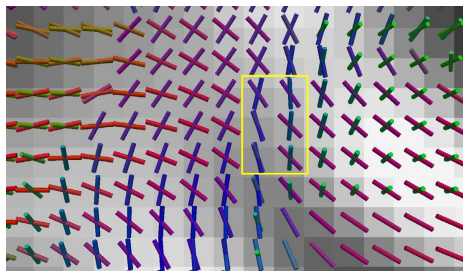

(c) Heuristic filtered

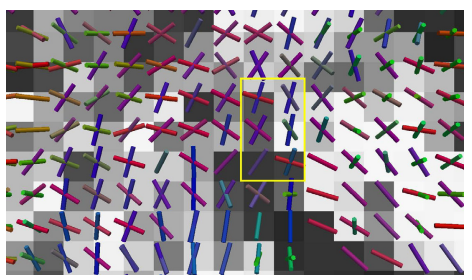

(b) Noised

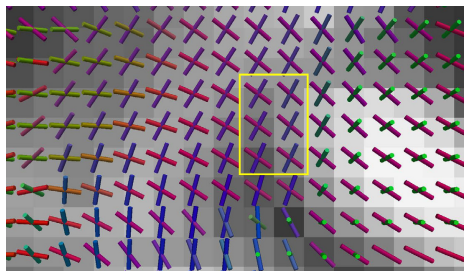

(d) Mixture-of-Watson filtered

Fig. 1. Gaussian filtering of noisy fibers and the results of the heuristic and mixture-ofWatson filtering. Voxels with particularly poor heuristic performance are highlighted in yellow.

\section{$3.2 \quad$ Filtering Experiment}

In our first experiment, we tested the effect of Gaussian filtering in the presence of noise for a single subject. We introduced varying levels of Gaussian noise in the volume fractions and orientations $\left(\Delta \sigma_{f}=0.01\right.$ and $\left.\Delta \sigma_{v}=0.05\right)$, followed by renormalization. We then filtered with weights defined by a Gaussian kernel ( $\sigma=2 \mathrm{~mm}, 5$ voxel support) with the heuristic, k-means, and Expectation Maximization approaches. We computed the error $E$ between the source and each of the noised and filtered volumes at each noise level:

$$
\begin{aligned}
E(A, B) & =\min \left(D\left(A_{0}, B_{0}\right)+D\left(A_{1}, B_{1}\right), D\left(A_{0}, B_{1}\right)+D\left(A_{1}, B_{0}\right)\right) \\
D(x, y) & =\frac{\left(f_{x}+f_{y}\right)}{2} \frac{180}{\pi} \arccos \left(\left|\boldsymbol{v}_{x}^{T} \boldsymbol{v}_{y}\right|\right)
\end{aligned}
$$

given a pair of two fiber voxel models $A=\left\{A_{0}, A_{1}\right\}$ and $B=\left\{B_{0}, B_{1}\right\}$, where $D$ is the volume-weighted angular difference between a pair of compartments. Fig. 1 shows an example slice accompanied by the noised and filtered fibers. We found the heuristic approach failed to restore some crossings where fibers had roughly equal volume, as highlighted in Fig. 1. We found the proposed approach to have lower error than the heuristic approach across all noise levels, with the Expectation Maximization approach outperforming the k-means approach, as shown in Fig. 2 Our serial implementation ran in five minutes on an Intel i5 $2.6 \mathrm{GHz}$ machine with $8 \mathrm{~GB}$ of RAM and typically converged in fewer than 10 iterations. 


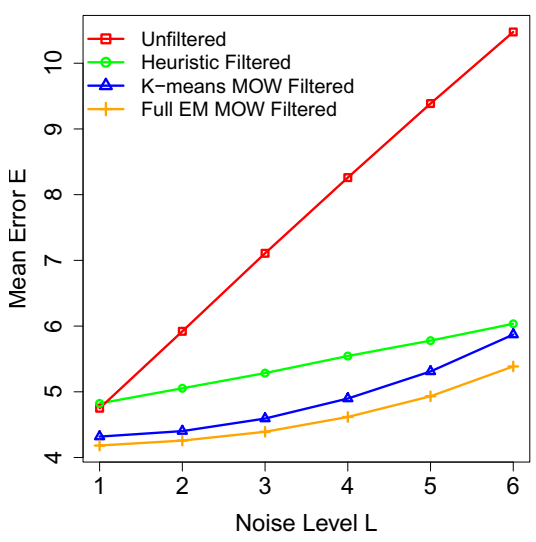

Fig. 2. The mean error as a function of noise level and type of filtering. Shown is the error for unfiltered (red square), heuristic (green circle), k-means (blue triangle), and full Expectation Maximization mixture-of-Watsons (orange cross) filtered volumes.

\subsection{Atlasing Experiment}

In our second experiment, we constructed a tractography atlas from the 80 subject population. Volumes were resampled into atlas space using both heuristic and mixture-of-Watsons interpolation with reorientation by finite strain rotation $\mathbf{J} / \sqrt{\mathbf{J J}^{T}}$ [11. Both cases used the same spatial transform computed with DTITK. Following this, the co-registered fibers were averaged using each method. Results are shown in Fig. 3. In our implementation, the interpolation took five minutes per subject, and population averaging took five minutes in total.

Deterministic streamline tractography was performed with both interpolation approaches with weights chosen by trilinear interpolation. Single tensor tracking was also performed for comparison. Multiple directions were included similarly to 11. Termination criteria were a volume fraction of 0.10 and angle of $35^{\circ}$. Other tracking parameters include step size of $1.5 \mathrm{~mm}$, one seed per voxel, and Runge-Kutta integration. Our serial tracking implementation took 10 minutes for the given parameters.

Major bundles were manually segmented using slice-based masks in TrackVis (www.trackvis.org), and included the corpus callosum, corona radiata, interior fronto-occipital fasciculus (IFOF), uncinate fasciculus, interior longitudinal fasciculus, and several portions of the superior longitudinal fasciculs (SLF). We compared the mean length and number of curves in each tract. We found close agreement among methods except the following. The two dorsal portions of the SLF were not present in the single tensor atlas, and the heuristic SLF had 50\% fewer curves and $40 \%$ shorter length than the mixture-of-Watsons SLF. The mixture-of-Watsons IFOF had $50 \%$ fewer curves but equal length to both other methods. The corpus callosum in the single tensor was similar to the heuristic reconstruction, but the mixture-of-Watsons reconstruction contained numerous anatomically plausible crossing fibers. These results are illustrated in Fig. 3. 


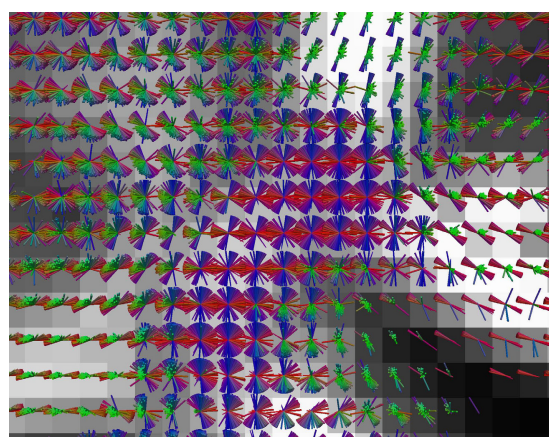

(a) Registered population fibers

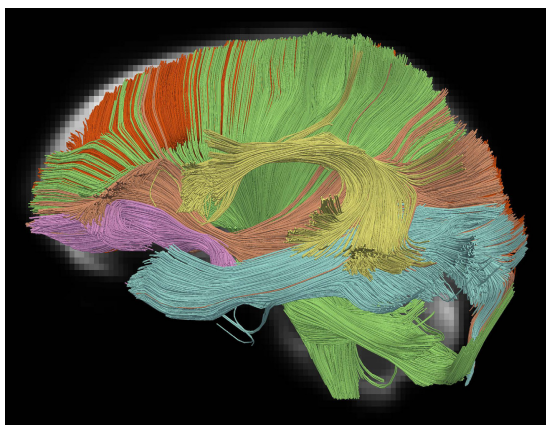

(c) Single tensor atlas

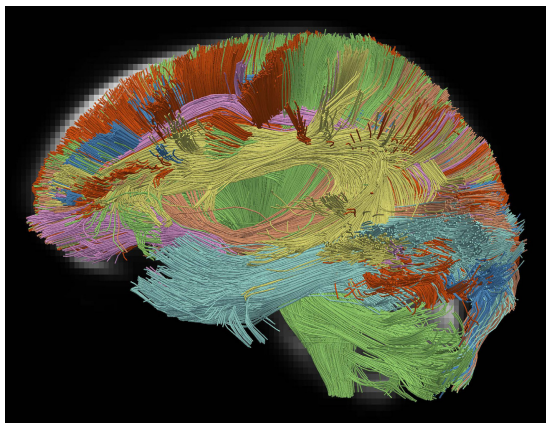

(e) Mixture-of-Watsons atlas

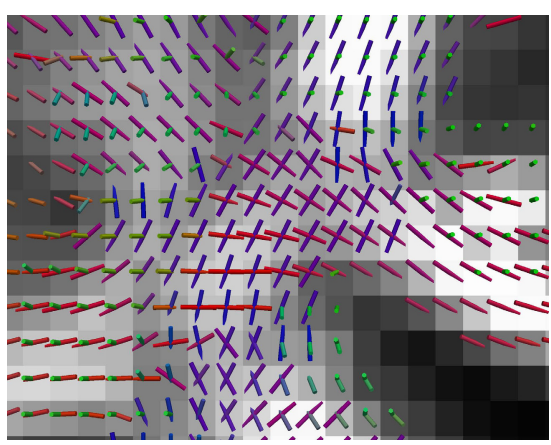

(b) Mixture-of-Watsons average fibers

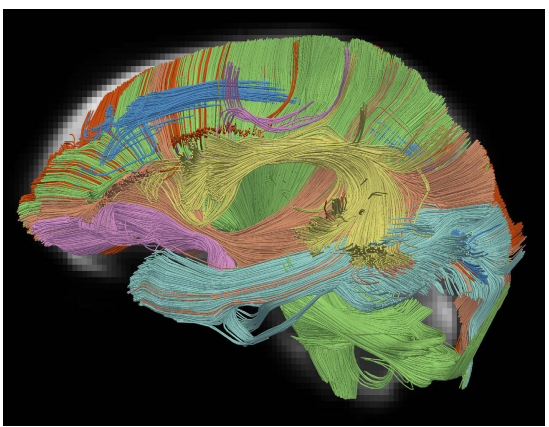

(d) Heuristic atlas

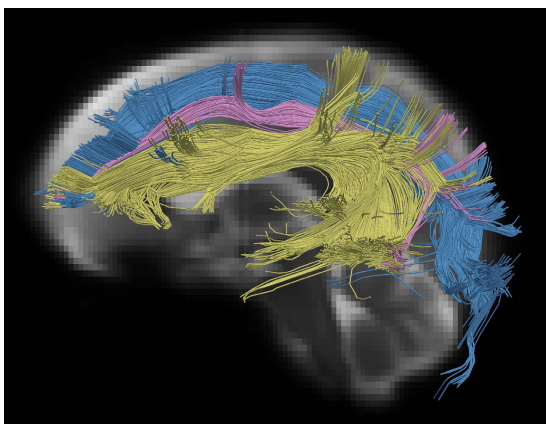

(f) Mixture-of-Watsons SLF tracts

Fig. 3. Construction and virtual dissection of atlas space tractography using the single tensor, heuristic, and mixture-of-Watsons estimation methods. Shown are examples of co-registered multi-fiber models (a) and their mixture-of-Watsons average (b). Also shown are tracks derived from a single tensor model (c), multi-fiber heuristic (d), and multi-fiber mixture-of-Watsons (e) approaches. The most significant strengths of the proposed method were in the superior longitudinal fasciculus, shown in (f), and corpus callosum (not shown). All cases used spatial transforms computed with DTI-TK from an 80 subject normal population. 


\section{Discussion and Conclusions}

We presented a method for performing filtering, interpolation, and averaging of multi-fiber ball-and-stick diffusion models using mixture-of-Watsons clustering. We found the presented Expectation Maximization algorithm to efficiently learn the mixture model parameters, and our experiments to demonstrate the value of the approach in several common volume analysis applications. Our first experiment suggests that the filtering method reduces error introduced by orientation noise. Our second experiment showed that our approach allows the reconstruction of white matter pathways across a large population, and the method improved the reconstruction of several major bundles, including the superior longitudinal fasciculus and corpus callosum. Notable limitations are that the diffusivity is not handled, the number of mixture components must be specified explicitly, and other reorientation strategies have not been explored. In conclusion, we find our approach to be an efficient and effective way to estimate multi-fiber ball-and-stick diffusion volumes resulting from a number of image analysis operations.

\section{References}

1. Bergmann, O., Kindlmann, G., Peled, S., Westin, C.F.: Two-tensor fiber tractography. In: 4th IEEE International Symposium on Biomedical Imaging: From Nano to Macro, ISBI 2007, pp. 796-799. IEEE (2007)

2. Behrens, T., Berg, H.J., Jbabdi, S., Rushworth, M., Woolrich, M.: Probabilistic diffusion tractography with multiple fibre orientations: What can we gain? Neuroimage 34(1), 144-155 (2007)

3. Taquet, M., Scherrer, B., Benjamin, C., Prabhu, S., Macq, B., Warfield, S.K.: Interpolating multi-fiber models by Gaussian mixture simplification. In: 2012 9th IEEE International Symposium on Biomedical Imaging (ISBI), pp. 928-931. IEEE (2012)

4. Rathi, Y., Michailovich, O., Shenton, M.E., Bouix, S.: Directional functions for orientation distribution estimation. Medical Image Analysis 13(3), 432-444 (2009)

5. Cook, P.A., Alexander, D.C., Parker, G.J.: Modelling noise-induced fibreorientation error in Diffusion-Tensor MRI. In: 2004 IEEE International Symposium on Biomedical Imaging: Nano to Macro, pp. 332-335. IEEE (2004)

6. Malcolm, J.G., Michailovich, O., Bouix, S., Westin, C.F., Shenton, M.E., Rathi, Y.: A filtered approach to neural tractography using the Watson directional function. Medical Image Analysis 14(1), 58 (2010)

7. Schwartzman, A., Dougherty, R.F., Taylor, J.E.: Cross-subject comparison of principal diffusion direction maps. Mag. Res. Medicine 53(6), 1423-1431 (2005)

8. Yap, P.T., Gilmore, J.H., Lin, W., Shen, D.: Poptract: population-based tractography. IEEE Transactions on Medical Imaging 30(10), 1829-1840 (2011)

9. Watson, G.S.: Distributions on the circle and sphere. Journal of Applied Probability, 265-280 (1982)

10. Sra, S., Jain, P., Dhillon, I.: Modeling data using directional distributions: Part II. Technical Report TR-07-05, Department of Computer Science, University of Texas at Austin, Austin, Texas (2007)

11. Zhang, H., Yushkevich, P.A., Alexander, D.C., Gee, J.C., et al.: Deformable registration of Diffusion Tensor MR images with explicit orientation optimization. Medical Image Analysis 10(5), 764-785 (2006) 